\title{
DESAFÍOS EN EDUCACIÓN SUPERIOR PARA LAS CIENCIAS SOCIALES
}

\section{CHALLENGES OF THE SOCIAL SCIENCES IN HIGHER EDUCATION DESAFIOS DAS CIÊNCIAS SOCIAIS NO ENSINO SUPERIOR}

\author{
Por: CORREA CRUZ - Lucelly, FORERO MENDOZA - Alexandra
}

\begin{abstract}
Magister en Ciencias de la Educación. Docente Facultad Ciencias Económicas y Administrativas, Universidad de la Amazonia. E-mail: l.correa@udla.edu.co, Colombia.

Maestría en Desarrollo sostenible y medio ambiente. Docente Programa Administración Financiera a Distancia, Universidad de la Amazonia. E-mail: a.forero@udla.edu.co, Colombia.
\end{abstract}

\section{RESUMEN}

En aras de generar trasformaciones sociales y personales, la educación no puede ser limitada a procesos, estructuras que solo son el reflejo de decisiones políticas, económicas y mercadológicas; un verdadero cambio social requiere políticas de educación que no estén plasmadas y enmarcadas desde escritorios ajenos a nuestra realidad.

De acuerdo con lo mencionado, el cuestionamiento y la reflexión principal en este escrito, radica en considerar que las ciencias sociales al estar en contacto con situaciones inherentes a la cotidianidad, deben fortalecer sus propuestas, sus actores e investigadores para proyectar argumentos sólidos hacia la transición paradigmática, y la transformación social.

Palabras clave: Educación, Ciencias Sociales, Investigación.

JEL: 123 


\begin{abstract}
If we are looking for social and personal transformation, education can not be limited to processes, structures that are only a reflection of political, economic and market related decisions; a real social change requires education policies that are not reflected and framed from desks outside our reality.

According to the above, the questioning and the main reflection at this writing, lies in considering the social sciences to be in contact with inherent in everyday situations, should strengthen their proposals, actors and researchers to project strong arguments to a paradigmatic transition, and social transformation.
\end{abstract}

Keywords: Education, Social Sciences, Research.

JEL: 123

\title{
RESUMO:
}

Com o fim de gerar transformações sociais e pessoais, a educação não pode ser limitada a processos, a estruturas que são apenas o reflexo das decisões políticas, econômicas e mercadológicas; uma verdadeira mudança social requer políticas de educação que não são refletidas e formuladas a partir de escritórios fora de nossa realidade.

De acordo com o exposto, o questionamento e a principal reflexão enunciada na presente escrita, encontra-se em considerar que as ciências sociais ao estar em contato com situações inerentes à cotidianidade, devem reforçar as suas propostas, seus atores e pesquisadores para projetar fortes argumentos à transição paradigmática, e a transformação social.

Palavras-chave: Educação, Ciências Sociais, investigação.

JEL: 123 


\section{INTRODUCCIÓN}

Actualmente se reconoce la educación como un derecho que se ha complejizado porque el ámbito de la educación formal también abarca desde la educación informal hasta la educación para el trabajo y el desarrollo humano; de esta manera, se considera que las políticas educativas han de ser indagadas desde la perspectiva de una educación para todos, en la cual influyen múltiples dimensiones, como la integración y acuerdos económicos, el cambio demográfico, y las nuevas tecnologías.

En este sentido de acuerdo con Verger Antoni (2002), se identificó que en la década de los ochenta (80), en las ciencias sociales, la educación sufrió el paradigma del desarrollo amparado en la teoría del capital humano, de igual manera, se encontró que en la década de los noventa (90) la educación fue permeada por la hegemonía de la ideología y del programa político neoliberal, generando rupturas de coherencia entre el sentido y la acción, entre el discurso y la práctica, evidenciados en procesos educativos cada vez menos reflexivos.

Aun así, en lo que respecta a educación superior, según el informe de la Unesco (2015: 12), en la década de los 2000 "la educación, se expandió aceleradamente con una enorme heterogeneidad, este crecimiento fue inequitativo", favoreciendo los sectores de mayores ingresos y de zonas urbanas, aunado a lo mencionado, se plantea que a pesar de experimentarse importantes progresos en términos de desarrollo global, durante esta década, continuaron las dificultades ocasionadas por expansión, en detrimento de una educación de calidad.

Retomando lo expuesto, se considera que desde las ciencias sociales, se requiere buscar conjuntamente caminos y alternativas a todas las paradojas y contradicciones del presente, dado que "aprender, recordar y olvidar es una dinámica constante del ser humano en constante transformación”, Castellanos (2010:5). Por lo tanto, se considera que en Colombia, es necesario propiciar nuevas formas de asumir decisiones, propuestas investigativas que no estén ajenas a la comunidad y sujetos comprometidos que puedan ser evaluados bajo marcos de ética y moralidad.

En este orden de ideas, se requiere formular investigaciones sociales, cuyas problemáticas impliquen que el investigador reconozca y haga explícito su lugar de enunciación, clarificando los intereses y motivaciones que guían su preocupación por el tema, y sus aportes tanto en el campo de conocimiento, como en el contexto social. Con lo dicho, se considera que los investigadores en ciencias sociales, deben asumir nuevos retos, de manera que sus estudios sean "pertinentes histórica, social, institucional y autorreferencialmente" (Jiménez y Torres, 2006: 17).

Por lo tanto, la perspectiva que se pretende plantear en este documento, se fundamenta en un pensar y actuar coherente, en un sentir personal y social, de manera que se aporten nuevos conocimientos desde las ciencias sociales, y por su puesto desde el compromiso y la responsabilidad de sus investigadores.

\section{Experiencias en Colombia y convergencias en Sur América}

En aras de iniciar la presente reflexión, se expondrán a continuación la situación actual del sistema educativo en Colombia y en algunos países de Sur América, en esta medida se plantearán algunas convergencias en cuanto a los cambios vivenciados en el sistema educativo. 
En Colombia, desde los años noventa, se han presentado varias denuncias sobre instituciones privadas, razón por la cual en el año 2001 el Ministerio de Educación Nacional inició el programa: Inspección y Vigilancia de la Educación Superior, pero la magnitud del trabajo y el escaso esfuerzo estatal permitieron que las irregularidades continuaran, posteriormente en el 2013, el Ministerio sancionó a 52 instituciones de educación superior e investigó 60 centros educativos que contaban con poco más de 360 mil matriculados, así mismo, en el 2014 según Acosta Silva; et al. (2015:223), "se organizó un debate sobre la continuidad de los problemas, a los que sumó contrataciones antiéticas con el estado”, y la realización de actividades con ánimo de lucro.

De igual manera, se ha identificado que en Suramérica, la situación es similar, por ejemplo en Venezuela, aunque se realizó un proceso de constituyente educativa y un conjunto de transformaciones más allá del concepto reformista mediante la misión Sucre, fundamentado en la inclusión, y a pesar de ser uno de los países en el mundo con más títulos universitarios según la Unesco, aún continúan los problemas en cuanto a calidad educativa, y la empleabilidad.

En este sentido, en el ámbito educativo de Brasil, se destaca que a pesar de los logros y avances, la calidad actualmente está decreciendo, y las diferentes expectativas de la población escolar, están incrementando.

Asimismo, en Paraguay, según la política educativa, denominada avances, deudas y desafíos, la educación media es gratuita y obligatoria, a pesar de ello hay un problema en cuanto a la cantidad de entidades de educación privada, lo cual es un indicador de mercantilización de la educación, por lo tanto, se considera que falta resolver el problema de oferta educativa de baja calidad, distinguiendo entre democratización y masificación de la educación.

Al respecto, se ha identificado que en Argentina existen problemáticas de producción de saberes socialmente productivos, respecto a lo cual Chicote y Dalmaroni (2008) plantean que lo fundamental ha sido la modificación de las condiciones de producción y transmisión de conocimiento, que han generado cambios y transformaciones evidenciadas en la proliferación de becas de investigación, de subsidios individuales y grupales, financiación de diversos tipos de proyectos, la publicación de tesis de doctorado, y de volúmenes colectivos que son producto del intercambio entre investigadores.

En relación con lo expuesto, desde Chile, Donoso Andrés (2015) comentó dada la presión económica, los elevados costos de matrículas, el endeudamiento y la deserción estudiantil por buscar trabajo, en el 2011 se organizó el movimiento estudiantil que inició el proceso de trasformación de la educación en Chile, para afrontar problemáticas con algunas becas de alimentación e irregularidades en la privatización de algunas universidades, es así que se concibe que desde este movimiento se incrementó el aporte a educación, se bajaron las tasas de algunos créditos, y según Sisto Vicente (2012: 44) en el ámbito de la educación pública, "se implementó la política de evaluación e incentivos al desempeño docente, y se incluyó un nuevo modelo de trabajo para el sector público”.

En consecuencia, se considera que, aunque en algunos países se ha ampliado cobertura, en otros se ha incrementado positiva o negativamente la inversión en educación, en Suramérica, no se ha logrado presentar mejoras evidentes en lo que 
concierne a la calidad educativa, la cual es endeble a pesar de acciones, protestas y movimientos en busca de generar transformaciones en el sistema educativo.

De igual manera, se encontró que existe el movimiento estudiantil o fuga de cerebros, puesto que la internacionalización no es suficiente, dado que la necesidad de postgrado hace que los estudiantes salgan a buscarlos como sea y los países más buscados en este sentido, son México, Argentina, Brasil, evidenciándose migraciones desde Chile, Colombia y Perú.

Adicionalmente, teniendo las situaciones expuestas, infiere que en la educación convergen dos tipos de fuerzas, por un lado lo público y por el otro lado lo privado, considerándose que históricamente se ha buscado un cambio educativo, particularmente en lo relacionado con la calidad en la educación, aunque con pocos avances.

\section{La transición paradigmática como escenario para generación de conocimientos}

La Universidad en el Siglo XXI, requiere que se rompa el blindaje disciplinario de las ciencias, desde la perspectiva de un vínculo común en cuanto a los problemas científicos, técnicos, humanísticos, en el ámbito educativo y en la relación con el entorno, es decir con el medio ambiente, por lo tanto, si estos problemas ya están identificados, la pregunta es cómo articularnos con las soluciones.

En relación con lo mencionado, se proponen los vectores transdisciplinarios, como constituyentes de nuevos paradigmas para desarrollar estrategias que lleguen más allá de las fronteras nacionales lo que implica una movilidad obligatoria de los estudiantes y repercute en la descolonización universitaria, siendo necesario atravesar escalas geográficas, trabajando lo multiregional desde la complementariedad.

En este sentido, se considera es imperante convocar a los estudiantes para construir investigación en el proceso formativo, para cimentar las bases educativas requeridas en la educación superior, teniendo a Comas y Lastra (2011), la labor académica requiere que quien lo cultiva resista al estrés de verse constantemente rebasado por los avances en su ciencia, consciente de que sabe cada vez más de cada vez menos.

De igual manera, se considera que la educación debe ir más allá de la tiza, el marcador, el lápiz, y el aula de clase, cuestionándonos sobre el impacto generado a nivel contextual y ambiental para proyectar el impacto esperado. En este orden de ideas, de acuerdo con Bernardo Mançano, (2008: 23), se considera necesario, un estilo de pensamiento desde un debate paradigmático o estilos de ver el mundo, afirmando que "aún falta una investigación adecuada y un análisis de los problemas y atolladeros generados, implicando nuevos elementos y nuevos desafíos".

Por lo tanto, se concibe que esas formas de ver la vida, producen discursos, cambian la realidad proponiéndose un debate entre dos visiones para discutir cómo se dan las relaciones de poder, como interpretamos la realidad, de manera tal, que por una parte se identifica la visión del desarrollo capitalista y por otra la lucha contra la desigualdad y la búsqueda de las necesidades del pueblo.

Estas tendencias han generado políticas de desarrollo, y teoría en aras de cambiar la realidad, las dos tendencias analizan el mismo proceso desde dos fines. La pregunta es, si será posible pensar el desarrollo a partir de otras relaciones sociales. 
De acuerdo con lo expuesto, los paradigmas que afectan la comprensión y producción del conocimiento están relacionados con el poder, evidenciándose la tensión entre quienes lo tienen y entre quienes solo lo ven desde abajo. Y ello de acuerdo con Clímaco (2012: 8) no podría ser casual, "incluso cuando el patrón colonial de poder no puede trazar el modo concreto en que las relaciones sociales ocurren, las controla en amplia medida, imponiéndoles un rumbo muy coincidente al de sus intereses”.

Teniendo en cuenta lo expuesto, se retoman los planteamientos de Méndez (2012: 63) quien afirma que es necesario:

Hablar de un "pensamiento alternativo que permita romper con la lógica monocultural del saber científico como único conocimiento válido, ajeno a otras epistemes no-científicas, populares, tradicionales, indígenas, campesinas, urbanas y locales”, postulándose la descolonialidad como un proceso complejo que pretende superar las huellas dejadas por la imposición del proyecto moderno occidental.

Según lo expuesto, se evidencia la necesidad de comprender la diversidad de la realidad intercultural y se plantea la descolonización de paradigmas para asumir convivencias de saberes, para visualizar, de construir y fomentar una sociedad de no exclusión, sin desposeídos o desolados, donde se opte por la filosofía del buen vivir, en aras de practicar el bien común, y la metacognición de nuestras representaciones sociales como aporte para generación de nuevos conocimientos y la construcción de un mundo mejor.

\section{Retos y Desafíos para las Ciencias Sociales en Colombia}

La universidad pública está sometida a gestionar las tensiones y reducir la multiplicidad de conflictos que sobrecargan. Acosta et al. (2015: 19), en este sentido, se considera relevante asumir "transformaciones estructurales y coyunturales", tanto al interior del ámbito universitario, como al exterior, en relación con las políticas de estado, mercados locales, globales y demandas de la sociedad civil.

Al respecto, se concibe que los múltiples desafíos de la educación superior son los siguientes: Minimizar el protagonismo financiero, generar capacidad para producción científica y tecnológica y conectarse con las necesidades de desarrollo de sus sociedades (Unesco, 2015), es decir investigando sobre los problemas educacionales de las mayorías, apoyando al mejoramiento y la reforma escolar.

En consecuencia, los principales retos y desafíos de la educación superior en Colombia, serían los siguientes:

No usar la represión para la solución de conflictos, abordando la educación desde una mirada crítica, teniendo en cuenta todo aquello que ha golpeado la educación pública y que ha acelerado la privatización, asumir la universidad mediante la construcción de modelos en red, proyectos, programas de investigación, construcción de conocimientos e intelectualidad crítica con presencia nacional e internacional, con plataformas de aprendizaje mundiales, desde una perspectiva liberadora, con currículos innovadores. 
Implementar reformas en las que primen el derecho humano, individual y social, antes que la ganancia, de manera que la ciencia no se someta a necesidades del desarrollo inmediato, sino que permita el intercambio de saberes, que fortalezcan la universidad, para afrontar cambios, y propiciar transformaciones que permeen en las políticas y sistemas educativos.

Ampliar los escenarios de participación de la educación superior, en aras de lograr mayor inclusión y democracia social, y conciencia ambiental, puesto que la educación superior al formar profesionales, debe contribuir al mejoramiento de la educación, la cultura, las comunidades, la sociedad y el medio ambiente.

Incluir programas de formación para los investigadores, respecto a lo cual de acuerdo con Yaber, De la vega y Guarino (2014: 19), si se agotan las fuentes de financiación internas, se pueden optar por fuentes de financiación externas como las planteadas en la Ley Orgánica de Ciencia, Tecnología e Innovación.

Adicionalmente, se considera relevante contribuir a los procesos de capacitación en programas de bilingüismo, lo cual según Castellanos, Juan (2010: 5) permitirá retomar estudios interculturales, y recrear en términos académicos e investigativos, en acciones que permitan encontrar los caminos para una América Latina, más humana, sabia e integrada.

Así mismo, no se puede seguir promoviendo los modelos excluyentes, siendo necesario incluir la educación del campo para pensar en la no salida de jóvenes y adultos a las ciudades, con proyectos educativos y didácticas, contextualizadas, que estén adecuadas a las necesidades de cada comunidad.

En este sentido se plantea la necesidad de políticas de inclusión en programas universitarios para minorías, de manera que no solo ingresen, sino que permanezcan en la universidad. Por lo tanto, se requieren incentivos, como becas de estudio, que propendan por la disminución de la desigualdad social, con el fin de conciliar la expansión, y la inclusión social con calidad, una calidad que no puede limitarse a replicar conocimientos y que no puede estar ajena a la realidad social.

En consecuencia, se considera que es reto de la educación superior, establecer relación equitativa, entre número de estudiantes, infraestructura, presupuesto, investigación y contexto socio ambiental, de esta forma según García M., Gabriel, en Hernández, Mario (2015), Colombia, "requiere una educación desde la cuna hasta la tumba, inconforme y reflexiva, que nos inspire un nuevo modo de pensar y nos incite a descubrir quiénes somos en una sociedad que se quiera más a sí misma”.

\section{La investigación como desafío para transformaciones sociales}

En lo que respecta a políticas de educación superior, se considera relevante que el investigador participe como sujeto constructor técnico científico, en la producción de conocimiento desde las ciencias sociales en la educación superior.

De esta manera, de acuerdo con Blesler (2008: 7), se considera que "producir conocimientos no puede concebirse ya como un derecho a conquistar, sino como una obligación a la cual sólo puede renunciarse desde la mala fe", por ende la investigación debe ser una prioridad educativa, como reto principal para la transformación social, con investigaciones no sesgadas por los avatares de la modernidad. En esta óptica, se plantea que "los procesos que conducen a la degradación 
del trabajo investigativo, no son el corolario de determinismos técnicos sino del resultado final de una cadena de decisiones políticas”.

Lo mencionado, teniendo en cuenta que la consecución de investigaciones se ve afectada desde la financiación, que por lo general es patrocinada por gobiernos condonados por grupos de poder económico, por intereses del mercado, con lo cual, se considera de acuerdo con Lens, José y Di Marco, María (2001: 7), que la investigación no puede ser degradada, ni limitada a "criterios de productividad o eficiencia”, ni a los ranking a escala mundial, como tampoco al lucro de sistemas productivos y mercadológicos.

De acuerdo con lo expuesto, se considera relevante un respaldo mayor y que visibilice el trabajo de los investigadores, que por lo general, dividen su tiempo entre el medio laboral, y sus estudios. En este sentido, según García (2007: 224) al asumir la investigación como reto en la educación superior, re requiere proponer alternativas de transformación, "desde la visión de sujetos colectivos en los procesos de formación, para abandonar las nociones instrumentalistas, examinando los efectos de las formas del poder y las dimensiones que permean los procesos de formación” y por lo tanto de investigación.

Así mismo y retomando los aportes de Fishman (2006: 80), se considera que es imperante la necesidad de incorporar "lo visual en los proyectos como un problema a investigar" (usando imágenes como material para el análisis) o como una herramienta para obtener datos durante trabajos de campo. En este sentido, se plantea que es necesario generar y continuar con alternativas como Clacso, Scielo, Redalyc, Latindex que publican gratis con acceso abierto para mostrar visibilidad de trabajos investigativos, los cuales pueden ser difundidos, por medio de consejos científicos, asociaciones, fundaciones.

Por ende, de acuerdo con Correa (1990: 34) se considera que el problema de investigación requiere la indagación de medios y mensajes como portadores de ciertos atributos relacionados directamente con el deseo de aprender, de observar, analizar e investigar, en aras de romper la limitación en la cualificación de la investigación, mediante estudios, más que diagnósticos, que proyecten como se ven las regiones y se planteen las alternativas de mejora sobre lo que esté ocurriendo en relación con lo investigado.

\section{A MANERA DE CONCLUSIÓN}

A través de nuestra historia, hemos transcurrido en lecturas del pasado y en dictámenes del presente, nuestro trasegar ha sido marcado por huellas de protesta, y movimientos de quienes en medio de las tensiones generadas por diversos tipos de poder, han tenido que decir basta, aún así, muchos de los cambios que en ciencias sociales se han logrado, no han traslapado ni permeado lo superficial, las estructuras, los modelos, y la masificación de la educación.

Generalmente, cuestionamos, investigamos y criticamos, pero pocas veces nos movemos de nuestra zona de confort, se producen discursos con la misma propagación que la contaminación, pero al contrario de esta, no nos afectamos solo intentamos sacudir a los demás desde la poltrona, mirando el mundo por los retrovisores, produciendo conocimiento como si fuéramos fábricas, pero con nuestras manos limpias y bañadas de crítica. 
El pasado que nos une, nos enseña que debemos unir nuestros postulados, como comunidades sin fronteras, de manera que las ciencias sociales puedan aportar en procesos de transformación que impacten favorablemente nuestra sociedad, procesos que impliquen propuestas y cambios de fondo, que nos cuestionen y nos muevan como unidad sistémica, donde cada quien desempeña un rol realmente relevante por poco o muy reconocido que este sea.

Desde esta perspectiva, es necesario asumirnos como universidad y estar en alerta constante de nuestra cotidianidad para indagar y proponer alternativas que no solo beneficien nuestros intereses personales o profesionales, sino que beneficien nuestro origen, nuestro ser y nuestro entorno, de manera que los desafíos presentados no se queden reconocidos y olvidados.

Por lo tanto entre los desafíos más relevantes para las ciencias sociales en educación superior, con los que debemos comprometernos por nuestras generaciones futuras, es la investigación, para mejorar la calidad educativa, con nuevos escenarios de participación y programas de formación, en los que se presenten aportes a la inclusión, integración y por supuesto al sostenimiento del medio ambiente y por ende nuestra vida en el planeta.

Es el momento de parar de hacernos los desentendidos, de dar menores esfuerzos, esperar mejores resultados, de criticar sin aportar, es el momento de asumirnos como colectivo, y como unidad en aras de un futuro común, proyectando lo que tendremos, lo que seremos y en especial, repensándonos a nosotros mismos. En este sentido, la máxima tarea, debe ser la duda, pero una duda inquietante y propositiva, puesto que de seguir adormecidos, utilizando tan solo nuestro dedo índice, puede costarnos más que nuestra realidad y nuestros propios ideales.

Debemos solidarizarnos, convivir, y escuchar las diferencias, para construir un mundo mejor, desde nuestras ideas, por lo cual es relevante tomar conciencia de nuestras acciones y orientar nuestro camino hacia un futuro construido en sociedad hacia desempeños y esfuerzos colectivos, pues la educación no es un negocio, es el mayor estandarte cuya dimensión está más allá de lo visible.

Es necesario abrir los ojos para soñar y poner los pies en la tierra para lograr lo que soñamos, es necesario detenernos y darnos cuenta que ni el universo, ni nosotros somos infinitos, y que somos seres gregarios por naturaleza, seres que dependemos del otro y ese otro, por ende también es necesario que desde la ciencias sociales propendamos por minimizar la visión del despilfarro, del aquí y del ahora, de los mínimos dados y los máximos esperados, para proponer alternativas que aporten en la solución de tantos problemas identificados, puesto que solo así podremos detener una catástrofe buscada en el inconsciente colectivo y anunciada por el consciente y la racionalización de unos pocos.

En consecuencia, se requiere hacer una pausa en este acelerado caminar y observar a nuestro alrededor y a nosotros mismos, para reconocer nuestro pasado, aprender de nuestros errores, valorar nuestro presente y proyectar nuestro futuro, de manera que logremos generar propuestas sociales, que aporten a estrategias y políticas de desarrollo acordes a nuestro contexto y nuestra realidad y planes de seguimiento, verificación con sus respectivos correctivos en la marcha, para minimizar las críticas a consecuencias de decisiones adjudicadas a pocos pero desapercibidas por todos. 


\section{REFERENCIAS}

1. ACOSTA, Adrián [et al.], (2015). Los desafíos de la universidad pública en América Latina y el Caribe. Clacso. E-Book. Ciudad autónoma de Buenos Aires. Disponible en: http://biblioteca.clacso.edu.ar/clacso/posgrados/20150722114530/LosDesafiosDeLaUnivPublica.pdf.

2. BLESLER, Alejandro (2008). Producción de conocimiento e intervención: Acerca del trabajo social como práctica científica. Segundo foro Latinoamericano: "Escenarios de la vida social, el trabajo social y las ciencias sociales en el siglo XXI". Argentina. Disponible en: http://biblioteca.clacso. edu.ar/Argentina/siuma/20120726064717/ProduccionconocimientoTS.pdf.

3. CASTELLANOS, Juan Manuel (2010) Palabras de Juan Manuel Castellanos en la graduación de los nuevos Doctores del Doctorado en Ciencias Sociales, Niñez y Juventud del Centro de Estudios Avanzados en Niñez y Juventud del Cinde y la Universidad de Manizales. Representante de los graduandos del Doctorado en Ciencias Sociales, Niñez y Juventud. Colombia, en Revista Latinoamericana de Ciencias Sociales, Niñez y Juventud, Centro de Estudios Avanzados en Niñez y Juventud Manizales, Vol. 8, núm. 2, julio-diciembre, pp. 1227-1230. Disponible en: http://www.redalyc. org/articulo.oa?id=77315155034

4. CLÍMACO, Danilo (2012). Fútbol, hipermasculinidad y colonialidad del poder. Reflexiones sobre el asesinato de género perpetrado por el capitán del Flamengo. Revista Kula, Antropólogos del Altico Sur. Abril, núm 6, pp. 35-47. Disponible en: http://www.revistakula.com.ar/wp-content/ uploads/2013/12/Danilo-de-Assis-Climaco-Futbol-hipermasculinidad-y-colonialidad-del-poder.pdf

5. COMAS, Oscar y LASTRA, Rosalía (2011). Literatura sobre estímulos económicos al profesorado universitario mexicano y la institucionalidad que introyectan. Iztapalapa Revista de ciencias sociales y humanidades, núm. 71. Año 32, julio-diciembre, pp. 87-117. Disponible en: dialnet.unirioja. es/descarga/articulo/4328684.pdf..

6. CORREA, Delia (1990). El modelo simbólico interactivo en la investigación sobre medios de enseñanza. Compilado en revista Q.webs.ull. Disponible en: https://dialnet.unirioja.es/servlet/articulo?codigo=152572.

7. CHICOTE, Gloria y DALMARONI, Miguel (2008). El vendaval de lo nuevo. Literatura y cultura en la Argentina moderna entre España y América Latina (1880-1930) En revista Anclajes. Vol. 13, número 2. Rosario, Beatriz Viterbo Editora, 334 p.

8. DONOSO, Andrés (2015). Ponencia en la VII, Conferencia Latinoamericana y Caribeña de Ciencias Sociales. Clacso. Colombia. Del 9 al 13 de noviembre de 2015. Medellín, Colombia.

9. FISHMAN, Gustavo (2006). Las fotos escolares como analizadores en la investigación educativa. Revista educación y realidades. Vol. 31, núm. 2, Julio-diciembre. Pp. 79-94. Disponible en: http://www.redalyc.org/pdf/3172/Resumenes/Resumen_317227044006_1.pdf.

10. GARCÍA, Oscar (2007). Los sujetos colectivos en la formación del estado nacional colombiano, Reseña publicada en revista Historia y Sociedad. Colombia, pp. 175-230. Disponible en: http://www.bdigital.unal.edu.co/25913/1/23410-81494-1-PB.pdf.

11. HERNÁNDEZ A., Mario (Marzo 21 de 2015). El financiamiento de la Educación Superior. El Espectador. Párrafo 4, 1p. Colombia. Disponible en:

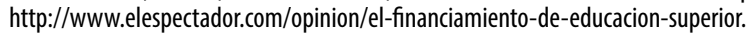

12. JIMÉNEZ., Absalón; TORRES C., Alfonso (2006). La práctica investigativa en Ciencias Sociales. DCS, Departamento de Ciencias Sociales. UPN, Universidad Pedagógica Nacional, Bogotá. Red de bibliotecas virtuales de ciencias sociales de América Latina y el Caribe de la red de centros miembros de Clacso. Colombia. ISBN: 958-8226-21-X. Disponible en: http://bibliotecavirtual.clacso.org.ar/ar/libros/colombia/dcsupn/practica.pdf.

13. LENS, José y DI MARC0, María (2001). Reflexiones en torno al papel de la escuela frente a la actual problemática del trabajo. Artículo presentado en el $5^{\circ}$. Congreso Nacional de estudios del trabajo. Del 1 al 3 de agosto. Aset. Asociación Argentina de Especialistas en Estudios del Trabajo. Disponible en: http://www.aset.org.ar/congresos/5/aset/PDF/LENS.PDF.

14. MANÇANO, Bernardo (2008). La ocupación como una forma de acceso a la tierra en Brasil: una contribución teórica y metodológica. Recuperando la tierra. El resurgimiento de movimientos rurales en África, Asia y América Latina. Sam Moyo y Paris Yeros [coord.]. Buenos Aires: Clacso, Consejo Latinoamericano de Ciencias Sociales. ISBN 978-987-1183-85-2 Disponible en: http://bibliotecavirtual.clacso.org.ar/ar/libros/sursur/ moyo/15Fernandes.pdf.

15. MÉNDEZ, R., Johan (2012). Eurocentrismo y modernidad. Una mirada desde la filosofía Latinoamericana y el pensamiento Descolonial. Redalyc. Red de revistas científicas de América Latina y el Caribe, España y Portugal. En revista Omnia. Año 18, №. 3. Pp. 49-65. Disponible en: http:// www.redalyc.org/html/737/73725513007/.

16. SISTO, Vicente (2012). Identidades Desafiadas: Individualización, Managerialismo y Trabajo Docente en el Chile Actual. Revista Psykhe. Vol. 21, núm. 2. Pp. 35-46. Disponible en: http://www.scielo.cl/pdf/psykhe/v21n2/art04.pdf.

17. UNESCO (2015). Situación educativa de América Latina y el Caribe: hacia la educación de calidad para todos al 2015. Informe diagnóstico del estado de avance de los países de América Latina y el Caribe. Disponible en: http://www.unesco.org/E941218C-E24D-4893-9C5D.

18. VERGER, Antoni (2002). Sistematización de experiencias en América Latina una propuesta para el análisis y la recreación de la acción colectiva desde los movimientos sociales, proyecto financiado por el Ministerio de Ciencia y Tecnología y el FEDER (proyectos I+D). Disponible en: http:// Www.alboan.org/archivos/353.pdf.

19. YABER, Guillermo; DE LA VEGA, Iván y GUARINO, Leticia (2014). Producción de conocimientos de países selectos en América Latina y del Caribe. Análisis comparativo, longitudinal y prospectivo en Ciencias Sociales y Humanidades de la Universidad Simón Bolívar. Artículo aceptado para publicación en la revista Mundo. Revista de Estudios Latinoamericanos. Disponible en: http://prof.usb.ve/gyaber/YaVeGudef.pdf. 